\title{
The determinants of malnutrition in the Irish elderly population: preliminary results from The Irish Longitudinal Study on Ageing (TILDA)
}

\author{
L. Bardon ${ }^{1,3}$, C.A. Corish ${ }^{2,3}$, M. Clarke ${ }^{1,3}$, K. Smuts ${ }^{2,3}$, L. Power ${ }^{2,3}$ and E.R. Gibney ${ }^{1,3}$ on \\ behalf of the MaNuEL Consortium \\ ${ }^{1}$ School of Agriculture and Food Science, University College Dublin, Dublin, Republic of Ireland, \\ ${ }^{2}$ School of Public Health, Physiotherapy and Sports Science, University College Dublin, Dublin, \\ Republic of Ireland and \\ ${ }^{3}$ UCD Institute of Food and Health, University College Dublin, Dublin, Republic of Ireland.
}

In Ireland, the proportion of the population aged 65 years or more is expected to rise from $11 \%$ in 2006 to $29 \%$ by $2041^{(1)}$. It is important that older people remain healthy, functional and independent for as long as possible ${ }^{(2)}$. Malnutrition has a detrimental effect on health, wellbeing, quality of life, disease outcome and dependency ${ }^{(3,4)}$. Determinants of malnutrition need to be identified to inform public health policy on its prevention and management. The Malnutrition in the Elderly (MaNuEL) project is conducting comparable analysis of longitudinal European datasets of older people to determine the predictors of nutritional risk. The aim of the current research was to assess the factors associated with developing malnutrition from baseline to 2-year follow-up using data from TILDA.

Data from the publicly accessible wave 1 and wave 2 TILDA datasets were used to conduct univariate regression analysis of a subcohort of participants $(n=1,841)$, using SPSS version 20.0 (IBM Corporation) to determine factors associated with malnutrition at 2-year follow-up. Malnutrition was defined as a BMI $<20 \mathrm{~kg} / \mathrm{m}^{2}$ and/or weight loss $\geqslant 10 \%$ at follow up. Exclusion criteria included being under 65 years of age, BMI or weight loss data unavailable at baseline and/or follow-up, and having malnutrition at baseline $\left(\mathrm{BMI}<20 \mathrm{~kg} / \mathrm{m}^{2}\right.$ or weight loss $\geqslant 10 \%$ over 2 years).

Approximately half the cohort was female $(50 \cdot 2 \%)$ with a mean age of 72 years. Determinants associated $(\mathrm{p}<0 \cdot 05)$ with malnutrition are displayed in the Table.

\begin{tabular}{|c|c|c|c|}
\hline & OR $(95 \% \mathrm{CI})$ & & OR $(95 \% \mathrm{CI})$ \\
\hline Older age & $1.04(1.01-1.07)$ & Polypharmacy ( $\geqslant 5$ medications) & $1.80(1.33-2 \cdot 42)$ \\
\hline Unmarried/divorced & $1.64(1.09-2.47)$ & Hospitalisation during 2 year follow up period & $1.63(1.17-2.27)$ \\
\hline Requiring social support & $2.50(1.47-4.28)$ & Poor self-rated health & $2.56(1.43-4.59)$ \\
\hline Low physical activity* & $1.54(1.07-2.22)$ & Cognitive impairment & $1.78(1.03-3.08)$ \\
\hline Difficulty walking & $2 \cdot 41(1 \cdot 56-3 \cdot 72)$ & Depressive symptoms & $1.65(1.01-2.71)$ \\
\hline Difficulty climbing stairs & $1.99(1.49-2.66)$ & & \\
\hline
\end{tabular}

*Low physical activity determined using the International Physical Activity Questionnaire.

Risk of malnutrition in TILDA is influenced by determinants from demographic, social, lifestyle, disease- related, physical functioning and psychological domains. In this analysis, gender, appetite, education, living situation, alcohol consumption, smoking, number of chronic diseases, pain, falls and handgrip strength were not associated with malnutrition. Future work will include multivariate regression analysis to establish the strongest independent determinants of malnutrition among the older Irish population.

This research is funded by the Department of Agriculture, Food and the Marine (DAFM) and the Joint Programming Initiative (JPI) 'Healthy Diet for a Healthy Life' (HDHL) under the Knowledge Hub on Malnutrition in the Elderly (MaNuEL) project.

1. McGill P. (2010) Illustrating Ageing in Ireland North and South: Key Facts and Figures. Belfast: Centre for Ageing Research and Development in Ireland.

2. Mathers JC. (2015) Br J Nutr. 113, 18-22.

3. Sullivan DH. (1995) Clin Geriatr Med. 11, 661-674.

4. Vellas BJ, Hunt WC, Romero LJ et al. (1997) Nutrition 13, 515-519. 